\title{
Power Spectra for Deterministic Chaotic Dynamical Systems
}

\author{
Ian Melbourne $^{*} \quad$ Georg A. Gottwald ${ }^{\dagger}$
}

23 July 2007. Revised 23 November 2007

\begin{abstract}
We present results on the broadband nature of power spectra for large classes of discrete chaotic dynamical systems, including uniformly hyperbolic (Axiom A) diffeomorphisms and certain nonuniformly hyperbolic diffeomorphisms (such as the Hénon map). Our results also apply to noninvertible maps, including Collet-Eckmann maps. For such maps (even the nonmixing ones) and Hölder continuous observables, we prove that the power spectrum is analytic except for finitely many removable singularities, and that for typical observables the spectrum is nowhere zero. Indeed, we show that the power spectrum is bounded away from zero except for infinitely degenerate observables.

For slowly mixing systems such as Pomeau-Manneville intermittency maps, where the power spectrum is at most finitely differentiable, nonvanishing of the spectrum remains valid provided the decay of correlations is summable.
\end{abstract}

\section{Introduction}

The power spectrum associated with a regular time series, stemming from a periodic or quasiperiodic underlying dynamical system, has discrete peaks at the harmonics and subharmonics. In contrast, the power spectrum of a chaotic signal is characterized by its broadband nature. The power spectrum has been successfully used by experimentalists to distinguish periodic, quasiperiodic, and chaotic motion [7].

Whereas the regular case with a spectrum consisting of $\delta$-peaks at discrete frequencies is easily understood, there are surprisingly few analytical results for the

*Mathematics and Statistics, University of Surrey, Guildford, Surrey GU2 7XH, UK. ism@math.uh.edu

${ }^{\dagger}$ Mathematics and Statistics, University of Sydney, NSW 2006, Australia. gottwald@maths.usyd.edu.au 
chaotic case. Most work has been done for mixing Axiom A systems [19] where the power spectrum is analytic apart from isolated singularities corresponding to RuellePollicott resonances [6].

In this paper, we elaborate on the broadband nature of the power spectrum in the chaotic setting. In particular, we show that for large classes of deterministic chaotic discrete dynamical systems, the power spectrum is analytic except for finitely many removable singularities, and is strictly positive.

Let $f: X \rightarrow X$ be a discrete dynamical system with ergodic invariant measure $\mu$. Given a square-integrable observable $v: X \rightarrow \mathbb{R}$, we define the power spectrum $S$ to be the square of the Fourier amplitudes of $v \circ f^{j}$ per unit time ${ }^{1}$

$$
S(\omega)=\lim _{n \rightarrow \infty} \frac{1}{n} \int_{X}\left|\sum_{j=0}^{n-1} e^{i j \omega} v \circ f^{j}\right|^{2} d \mu, \quad \omega \in[0,2 \pi] .
$$

For $\omega \in(0,2 \pi)$, the Wiener-Khinchin theorem [11] relates the power spectrum to the Fourier transform of the autocorrelation function according to

$$
S(\omega)=\sum_{k=-\infty}^{\infty} e^{i k \omega} \rho(k)
$$

where

$$
\rho(k)=\int_{X} v \circ f^{k} v d \mu-\left(\int_{X} v d \mu\right)^{2}
$$

is the autocorrelation function. This relation is valid for sufficiently rapidly decaying (eg. summable) autocorrelations. It is then immediate [19] that $S(\omega)$ is an analytic function if and only if the autocorrelations decay exponentially. (Typically, $S(0)=$ $S(2 \pi)=+\infty$ so strictly speaking $S(\omega)$ is analytic with removable singularities at 0 and $2 \pi$. The singularities disappear if and only if $\int_{X} v d \mu=0$.)

For definiteness, we introduce our main results in the context of the logistic map, or quadratic family, $f(x)=a x(1-x), 0 \leq a \leq 4$. It is well-known that there is a unique attractor $X \subset[0,1]$ for each value of $a$. For an open and dense set of parameters $X$ is a periodic orbit, but for a positive measure set of parameters $X$ is strongly chaotic with a unique absolutely continuous invariant measure $\mu[10,2]$ and moreover satisfies the Collet-Eckmann condition [4]: there exists $c>0, \lambda>1$ such that

$$
\left|D f^{n}\left(f\left(\frac{1}{2}\right)\right)\right| \geq c \lambda^{n} \text { for all } n \geq 1
$$

Then the Lyapunov exponent is positive, the attractor $X$ consists of finitely many intervals $X_{1}, \ldots, X_{q}$ permuted cyclically by $f$, and $f^{q} \mid X_{i}$ has exponential decay of

\footnotetext{
${ }^{1}$ Often $e^{i j \omega}$ is replaced by $e^{2 \pi i j \omega / n}$ in the literature, but this is just a rescaling of the domain.
} 
correlations for Hölder observables for each $i=1, \ldots, q[12,22]$. (By Lyubich [14], the two possibilities mentioned above, periodic or Collet-Eckmann, account for almost every parameter $a \in[0,4]$.)

Theorem 1.1 Fix a parameter $a \in[0,4]$ such that the Collet-Eckmann condition is satisfied, with the attractor $X$ consisting of $q$ intervals. Let $v:[0,1] \rightarrow \mathbb{R}$ be a Hölder continuous observable. Then

(i) There is an analytic function $\tilde{S}:[0,2 \pi] \rightarrow \mathbb{R}$ such that $S(\omega)=\tilde{S}(\omega)$ for all $\omega \neq 2 \pi j / q, j=0,1, \ldots, q$.

(ii) At $\omega=2 \pi j / q$, either $S(\omega)=\infty$ (the typical case) or $S(\omega)=\tilde{S}(\omega)$ (codimension one).

(iii) Except for infinitely degenerate ${ }^{2}$ observables $v$, there is a constant $c>0$ such that $S(\omega) \geq \tilde{S}(\omega) \geq$ c for all $\omega \in[0,2 \pi]$.

Remark 1.2 (a) Again we note that Theorem 1.1(i,ii) is well-known when $q=1$ (due to exponential decay of correlations and the Wiener-Khinchin theorem). For $q \geq 2$, parts (i) and (ii) are presumably well-known to experts in the field but we could not find a proof (or a precise statement of the result) in the literature. For completeness, we state and prove an appropriate generalisation of the Wiener-Khinchin theorem in the Appendix. Nevertheless, in the main part of the paper we give a different proof of parts (i) and (ii) for all $q \geq 1$ which has the advantage that it immediately yields a criterion for $\tilde{S}(\omega)=0$ (which is used in the proof of part (iii)).

Part (iii) of Theorem 1.1 seems to be new for all $q \geq 1$.

(b) The results in $[12,22]$ are stated more generally for observables of bounded variation. Theorem 1.1(i,ii) remains valid for such observables, but part (iii) uses Hölder continuity.

We also obtain results in the invertible setting. Suppose that $f: M \rightarrow M$ is an Axiom A (uniformly hyperbolic) diffeomorphism. Recall that an invariant set $X \subset M$ is called a (nontrivial) hyperbolic basic set if $X$ is transitive and locally maximal, and $X$ is not a periodic orbit. Such a basic set consists of $q$ components $X_{1}, \ldots, X_{q}$ permuted by $f$, and $f^{q} \mid X_{i}$ is topologically mixing for each $i$. Given any Hölder potential on $X$, there is a unique equilibrium measure $\mu$, and $\mu \mid X_{i}$ is mixing under $f^{q}$. Again it is well-known [3] that $f^{q} \mid X_{i}$ has exponential decay of correlations for Hölder observables.

\footnotetext{
${ }^{2}$ Lying in a closed subspace of infinite codimension within the Banach space of Hölder continuous observables
} 
Theorem 1.3 Suppose that $X$ is a basic set with $q$ components for an Axiom $A$ diffeomorphism $f: M \rightarrow M$. Let $\mu$ be an equilibrium measure for a Hölder potential. Let $v: M \rightarrow \mathbb{R}$ be a Hölder observable and define $S(\omega)$ as in (1.1). Then parts (i)-(iii) of Theorem 1.1 hold.

Our results extend to a large class of nonuniformly hyperbolic systems modelled by the tower construction of Young [23, 24]. In Young [23], exponential decay of correlations (for $f^{q}$ ) is proved for a large class of nonuniformly hyperbolic systems, including planar periodic billiards and Hénon-like diffeomorphisms. The conclusions of Theorem 1.1 hold for such systems.

Young [24] considers nonuniformly hyperbolic systems with slow decay of correlations. This includes Pomeau-Manneville intermittency maps with indifferent fixed points. Analyticity in Theorem 1.1 obviously breaks down for such systems. Nevertheless, we obtain again that the power spectrum is bounded away from zero except for infinitely degenerate observables, provided the decay of correlations is summable.

The remainder of the paper is organised as follows. In Section 2, we prove parts (i) and (ii) of Theorem 1.1 and give a criterion for zeroes in the power spectrum. In Section 3, we prove Theorem 1.3 and part (iii) of Theorem 1.1. (The order of proof is due to the fact that (i) and (ii) are simpler for noninvertible maps, whereas it is convenient to prove (iii) first for invertible maps.) In Section 4, we indicate the modifications required for nonuniformly hyperbolic systems with exponential and slow decay properties. In Section 5, we present some numerics illustrating our results for the logistic map.

\section{Proof of Theorem 1.1(i,ii) and a criterion for $\tilde{S}(\omega)=0$}

In this section, we prove analyticity of the power spectrum (parts (i) and (ii) of Theorem 1.1) and we give a criterion for the power spectrum to vanish. Throughout, we consider the logistic map $f(x)=a x(1-x)$ where $a \in[0,4]$ is such that the ColletEckmann condition is satisfied. Recall that the attractor $X \subset[0,1]$ consists of $q$ intervals permuted by $f$ such that $f^{q}$ has exponential decay of correlations for Hölder observables on each interval. We consider first the case $q=1$, and then extend to the case $q \geq 2$.

\section{The mixing case $q=1$}

First, we adapt ideas in [8] to decompose $v-\int_{X} v d \mu$ into a (twisted) coboundary $e^{i \omega} \chi_{\omega} \circ f-\chi_{\omega}$ which does not alter the value of $S(\omega)$ and a "martingale" $\tilde{v}_{\omega}$ for which 
the computation of $S(\omega)$ is trivial. (Our treatment closely follows [15].)

Let $\alpha \in(0,1)$. The space of Hölder continuous functions $C^{\alpha}(X)=\{u: X \rightarrow \mathbb{C}$ : $\left.|u|_{\alpha}=\sup _{x \neq y}|u(x)-u(y)| /|x-y|^{\alpha}<\infty\right\}$ is a Banach space when endowed with the norm $\|u\|_{\alpha}=|u|_{\infty}+|u|_{\alpha}$ where $|u|_{\infty}=\sup _{x \in X}|u(x)|$.

Let $U: L^{2}(X) \rightarrow L^{2}(X)$ be the Koopman operator $(U v=v \circ f)$ and let $L=$ $U^{*}: L^{2}(X) \rightarrow L^{2}(X)$ be the Perron-Frobenius operator. We have $L U=I$. The key property that we make use of is that if $u \in C^{\alpha}(X)$, then $\left\|L^{n} u-\int_{X} u d \mu\right\|_{\alpha}$ converges to zero exponentially quickly [23].

Lemma 2.1 Suppose that $v: X \rightarrow \mathbb{R}$ and that $v \in C^{\alpha}(X)$ for some $\alpha>0$. Then for all $\omega \in[0,2 \pi]$,

$$
v=\int_{X} v d \mu+e^{i \omega} \chi_{\omega} \circ f-\chi_{\omega}+\tilde{v}_{\omega}
$$

where (a) $\chi_{\omega}, \tilde{v}_{\omega} \in C^{\alpha}(X),(b) \omega \mapsto \chi_{\omega}, \tilde{v}_{\omega}$ are analytic on $[0,2 \pi]$, and (c) L $\tilde{v}_{\omega}=0$.

Proof Let $\hat{v}=v-\int_{X} v d \mu$. Since $\int_{X} \hat{v} d \mu=0$, the series $\sum_{j=1}^{\infty} e^{-i j \omega} L^{j} \hat{v}$ converges to a Hölder function $\chi_{\omega} \in C^{\alpha}(X)$. Note that $\chi_{\omega}$ depends analytically on $\omega$ since the Fourier coefficients $L^{j} \hat{v}$ decay exponentially quickly (in the Hölder norm).

Define the analytic family of Hölder functions $\tilde{v}_{\omega}=\chi_{\omega}-e^{i \omega} \chi_{\omega} \circ f+\hat{v}$. Then

$$
e^{-i \omega} L \tilde{v}_{\omega}=e^{-i \omega} L \chi_{\omega}-L U \chi_{\omega}+e^{-i \omega} L \hat{v}=\chi_{\omega}-\chi_{\omega}=0 .
$$

Hence, we obtain the required decomposition.

Lemma 2.2 Let $\tilde{S}(\omega)=\int_{X}\left|\tilde{v}_{\omega}\right|^{2} d \mu$. Then $\tilde{S}(\omega)$ is analytic, and $S(\omega)=\tilde{S}(\omega)$ for $\omega \in(0,2 \pi)$. Moreover, $S(0)=\tilde{S}(0)$ if $\int_{X} v d \mu=0 ; S(0)=\infty$ if $\int_{X} v d \mu \neq 0$.

Proof It is immediate that $\tilde{S}(\omega)=\int_{X}\left|\tilde{v}_{\omega}\right|^{2} d \mu$ is analytic. For $\omega \in(0,2 \pi)$, we have

$$
\sum_{j=0}^{n-1} e^{i j \omega} v \circ f^{j}=\left(1-e^{i \omega}\right)^{-1}\left(1-e^{i n \omega}\right) \int_{X} v d \mu+e^{i n \omega} \chi_{\omega} \circ f^{n}-\chi_{\omega}+\sum_{j=0}^{n-1} e^{i j \omega} \tilde{v}_{\omega} \circ f^{j}
$$

and so

$$
\left|\left\|\sum_{j=0}^{n-1} e^{i j \omega} v \circ f^{j}\right\|_{2}-\left\|\sum_{j=0}^{n-1} e^{i j \omega} \tilde{v}_{\omega} \circ f^{j}\right\|_{2}\right| \leq 2\left(1-e^{i \omega}\right)^{-1} \int_{X} v d \mu+2\left\|\chi_{\omega}\right\|_{2} .
$$

Hence, $S(\omega)=\lim _{n \rightarrow \infty} \frac{1}{n} \int_{X}\left|\sum_{j=0}^{n-1} e^{i j \omega} \tilde{v}_{\omega} \circ f^{j}\right|^{2} d \mu$, since the terms on the right hand side are bounded in $n$. In the remainder of the proof we omit the tilde's. The integral 
contains $n^{2}$ terms of the form $e^{i(j-k) \omega} \int_{X} v_{\omega} \circ f^{j} \bar{v}_{\omega} \circ f^{k} d \mu$ where $\bar{v}_{\omega}$ denotes complex conjugation. Now we use the fact that $L v_{\omega}=0$. If $j>k$,

$$
\int_{X} v_{\omega} \circ f^{j} \bar{v}_{\omega} \circ f^{k} d \mu=\int_{X} v_{\omega} \circ f^{j-k} \bar{v}_{\omega} d \mu=\int_{X} v_{\omega} L^{j-k} \bar{v}_{\omega} d \mu=0
$$

and similarly the cross-terms with $j<k$ vanish. The first equality follows from stationarity, the second from the definition of the Perron-Frobenius operator. By stationarity, the diagonal terms are all equal to $\tilde{S}(\omega)=\int_{X}\left|v_{\omega}\right|^{2} d \mu$. It follows that $S(\omega)=\tilde{S}(\omega)$ for $\omega \in(0,2 \pi)$. Moreover, the above calculation works for $\omega=0,2 \pi$ provided $\int_{X} v d \mu=0$. If this is not the case, then the first term in the right-hand-side of (2.2) is replaced by $n \int_{X} v d \mu$ which diverges to infinity.

Corollary 2.3 Let $\omega \in[0,2 \pi]$. Then $\tilde{S}(\omega)=0$ if and only if there is a continuous function $\chi: X \rightarrow \mathbb{C}$ such that

$$
v=\int_{X} v d \mu+e^{i \omega} \chi \circ f-\chi
$$

Proof By definition, $\tilde{S}(\omega)=0$ if and only if $\tilde{v}_{\omega} \equiv 0$, so the result follows from $(2.1)$ (taking $\chi$ to be the Hölder continuous function $\chi_{\omega}$ ).

\section{The nonmixing case $q \geq 2$}

Now suppose that $X$ consists of $q$ intervals permuted by $f$. Restricting to Hölder functions, the Perron-Frobenius operator $L$ has simple eigenvalues $e^{2 \pi i k / q}, k=$ $0,1, \ldots, q-1$, and the remainder of the spectrum lies strictly inside the unit circle. Let $v_{0}, \ldots, v_{q-1}$ be the corresponding eigenfunctions. (Note that $v_{0}=\int_{X} v d \mu$.) Then $v_{k} \circ f=e^{-2 \pi i k / q} v_{k}$. Write $v=v_{0}+\cdots+v_{q-1}+\hat{v}$. We have

$$
\sum_{j=0}^{n-1} e^{i j \omega} v \circ f^{j}=\sum_{k=0}^{q-1}\left(1-e^{i(\omega-2 \pi k / q)}\right)^{-1}\left(1-e^{i n(\omega-2 \pi k / q)}\right) v_{k}+\sum_{j=0}^{n-1} e^{i j \omega} \hat{v} \circ f^{j}
$$

Provided $\omega \neq 2 \pi k / q$ for $k=0, \ldots, q$, we can disregard the terms $v_{0}, \ldots, v_{q-1}$ when computing $S(\omega)$. Once again, the series $\sum_{j=1}^{\infty} e^{i j \omega} L^{j} \hat{v}$ converges exponentially quickly to a Hölder observable $\chi_{\omega}$ depending analytically on $\omega$. Proceeding as before, we find that $S$ agrees with an analytic function $\tilde{S}$ except possibly for singularities at $2 \pi k / q$ for $k=0, \ldots, q .\left(S(2 \pi k / q)=+\infty\right.$ if and only if $v_{k} \neq 0$.) Moreover, $\tilde{S}(\omega)=0$ if and only if the criterion (2.3) is satisfied for some continuous function $\chi: X \rightarrow \mathbb{C}$. 


\section{Axiom A systems}

For hyperbolic basic sets and Hölder observables, exponential decay of correlations (for $f^{q}$ in the nonmixing case) is often obtained by (a) passing to a subshift of finite type, (b) quotienting by stable directions to obtain a noninvertible map, (c) proving exponential decay of the Perron-Frobenius operator $L$ for the noninvertible map. See for example $[3,17]$.

For our purposes, only step (b) requires further discussion. The standard argument of $[20,3]$ shows that $v=v^{\prime}+\chi^{\prime} \circ f-\chi^{\prime}$ where $v^{\prime}$ depends only on future coordinates and so is well-defined on the quotient noninvertible system. Moreover, $v^{\prime}$ and $\chi^{\prime}$ are Hölder (with different exponents from $v$ ). We require instead that $v=v_{\omega}^{\prime}+e^{i \omega} \chi_{\omega}^{\prime} \circ f-\chi_{\omega}^{\prime}$ so that the $\chi_{\omega}^{\prime}$ terms can be ignored in computing $S(\omega)$. This is achieved by making obvious modifications to the arguments in $[20,3]$. First, for each $x$ in $X$, choose $\hat{x} \in X$ such that the operation $x \mapsto \hat{x}$ depends only on future coordinates and $(\hat{x})_{n}=x_{n}$ for all $n \geq 0$. Define $\chi_{\omega}^{\prime}(x)=\sum_{j=0}^{\infty} e^{i j \omega}\left(\hat{v}\left(f^{j}(x)\right)-\hat{v}\left(f^{j}(\hat{x})\right)\right.$. By exponential contraction along stable manifolds, this is an analytic Fourier series. This uniquely specifies $v_{\omega}^{\prime}$ and a direct calculation shows that $v_{\omega}^{\prime}(x)=\sum_{j=0}^{\infty} e^{i j \omega} \hat{v}\left(f^{j}(\hat{x})\right)-\sum_{j=0}^{\infty} e^{i(j+1) \omega} \hat{v}\left(f^{j}(\widehat{f x})\right)$ so that $v_{\omega}^{\prime}$ depends only on future coordinates. The usual argument shows that $\chi_{\omega}^{\prime}$ is Hölder (with a different exponent) and it follows that $v_{\omega}^{\prime}$ is Hölder.

By this construction, if $S(\omega)=0$ for some $\omega \in[0,2 \pi]$, then criterion (2.3) is valid as before, with $\chi$ replaced by $\chi+\chi^{\prime}$. Hence we may reduce to the noninvertible case by carrying out steps (a) and (b). By step (c), the methods in Section 2 for the logistic map go through unchanged. It follows that we obtain parts (i) and (ii) of Theorem 1.3, and the criterion (2.3) for vanishing spectrum.

To prove that the spectrum is typically nonvanishing, we make use of the fact that there is an abundance of homoclinic points. More precisely, there is a countable infinity of periodic points in $X$. For each periodic point $x_{0} \in X$ of period $p$, there exists a homoclinic point $x_{1} \in X$, distinct from $f^{j}\left(x_{0}\right)$ for all $j$, such that $f^{n p}\left(x_{1}\right) \rightarrow x_{0}$ as $n \rightarrow \pm \infty$. For each of these infinitely many homoclinic points, we associate a degeneracy condition that is automatically satisfied if the spectrum vanishes.

Here, we closely follow [16]. First suppose for simplicity that $x_{0} \in X$ is a fixed point (in particular, $p=q=1$ ). Let $x_{1} \in X$ be homoclinic to $x_{0}$ (so $x_{1} \neq x_{0}$ and $f^{n}\left(x_{1}\right) \rightarrow x_{0}$ as $\left.n \rightarrow \pm \infty\right)$. Define

$$
g(\omega)=\sum_{j=-\infty}^{\infty} e^{i j \omega}\left(v\left(f^{j}\left(x_{1}\right)\right)-v\left(x_{0}\right)\right) .
$$

Lemma 3.1 The function $g:[0,2 \pi] \rightarrow \mathbb{C}$ is analytic and if $\tilde{S}(\omega)=0$ then $g(\omega)=0$. 
Proof Due to exponential contraction/expansion along stable/unstable manifolds, $g$ is a Fourier series with exponentially decaying coefficients and hence is analytic on $[0,2 \pi]$.

Suppose that $\tilde{S}(\omega)=0$ for some $\omega$. We assume that $\omega \in(0,2 \pi)$ since the argument for $\omega=0,2 \pi$ is slightly different (but easier). Let $\hat{v}=v-\int_{X} v d \mu$. By (2.3),

$$
\sum_{j=-n}^{n-1} e^{i j \omega} \hat{v}\left(f^{j}\left(x_{1}\right)\right)=e^{i n \omega} \chi\left(f^{n}\left(x_{1}\right)\right)-e^{-i n \omega} \chi\left(f^{-n}\left(x_{1}\right)\right) .
$$

Also, by $(2.3), \hat{v}\left(x_{0}\right)=\left(e^{i \omega}-1\right) \chi\left(x_{0}\right)$ and so

$$
\begin{aligned}
\sum_{j=-n}^{n-1} e^{i j \omega}\left(v\left(f^{j}\left(x_{1}\right)\right)-v\left(x_{0}\right)\right) & =\sum_{j=-n}^{n-1} e^{i j \omega}\left(\hat{v}\left(f^{j}\left(x_{1}\right)\right)-\hat{v}\left(x_{0}\right)\right) \\
& =e^{i n \omega}\left(\chi\left(f^{n}\left(x_{1}\right)\right)-\chi\left(x_{0}\right)\right)-e^{-i n \omega}\left(\chi\left(f^{-n}\left(x_{1}\right)\right)-\chi\left(x_{0}\right)\right) \\
& \rightarrow 0 \text { as } n \rightarrow \infty .
\end{aligned}
$$

Hence $g(\omega)=0$.

Proof of Theorem 1.3(iii) We continue to suppose that $X$ contains a fixed point $x_{0}$. Choose a second homoclinic point $x_{1}^{\prime}$ (lying on a distinct homoclinic orbit) to obtain a second analytic function $g^{\prime}(\omega)=\sum_{j=-\infty}^{\infty} e^{i j \omega}\left(v\left(f^{j}\left(x_{1}^{\prime}\right)\right)-v\left(x_{0}^{\prime}\right)\right)$. By Lemma 3.1, if $\tilde{S}$ vanishes somewhere, then $g$ and $g^{\prime}$ have a common zero.

Since $g$ is analytic, either $g \equiv 0$ or $g$ vanishes at only finitely many points. The homoclinic point $x_{1}$ is isolated from the remainder of the homoclinic orbit $f^{n}\left(x_{1}\right)$, $n= \pm 1, \pm 2, \ldots$. Hence, we can make small changes to $g$ as desired by modifying the value of $v$ at $x_{1}$ in such a way that $v$ remains Hölder. Independently, we can make small changes to $g^{\prime}$ by modifying the value of $v$ at $x_{1}^{\prime}$.

If $g \equiv 0$, then after a small perturbation $g$ is nowhere zero and $\tilde{S}$ is nowhere zero by Lemma 3.1. It remains to consider the case when $g$, and similarly $g^{\prime}$, vanishes at finitely many points. In particular, $g$ and $g^{\prime}$ have at most finitely many common zeroes. Suppose that $\omega$ is a common zero. Then we can perturb $v$ at $x_{1}$ so that $g(\omega) \neq$ 0 . This might introduce new zeroes for $g$ but we can ensure that whenever $g(\omega) \neq 0$ and $g^{\prime}(\omega)=0$ before perturbation (there are only finitely many such $\omega$ ), then this situation persists after perturbation. Hence no new common zeroes are introduced and so the number of common zeroes is reduced by one via this perturbation. After finitely many such perturbations, we reach the situation where there are no common zeroes.

The same arguments work with minor modifications if the fixed points are replaced by periodic points. (We now incorporate the case $q \geq 2$.) Suppose for example that $x_{0}$ 
is a periodic point of period $p$. Setting $V=\sum_{j=0}^{p-1} e^{i j \omega} v \circ f^{j}$ and $W=\sum_{j=0}^{p-1} e^{i j \omega} \chi \circ f^{j}$, and substituting into (2.3), we obtain $V=\int_{X} V d \mu+e^{i p \omega} W \circ f^{p}-W$. Let $x_{1}$ be homoclinic to $x_{0}\left(f^{n p}\left(x_{1}\right) \rightarrow x_{0}\right.$ as $\left.n \rightarrow \pm \infty\right)$. Replacing $f$ by $f^{p}$ and $\omega$ by $p \omega$ in the above argument, we obtain the analytic function $g(\omega)=\sum_{j=-\infty}^{\infty} e^{i j p \omega}\left(V\left(f^{j p}\left(x_{1}\right)\right)-\right.$ $\left.V\left(x_{0}\right)\right)$. Hence, by considering any pair of distinct periodic orbits, we obtain that typically criterion (2.3) is not satisfied for any $\omega \in[0,2 \pi]$. Since there are infinitely many periodic orbits in $X$, it follows that (2.3) is satisfied for some $\omega$ only in infinitely degenerate situations.

Proof of Theorem 1.1(iii) In the noninvertible case, the definition of homoclinic point must be modified. For example, given a fixed point $x_{0}$, we replace the homoclinic orbit $f^{n}\left(x_{1}\right)$ by a sequence $x_{n}, n \geq 0$, with $x_{1} \neq x_{0}$ such that $f\left(x_{n}\right)=x_{n-1}$ for $n \geq 1$ and $x_{n} \rightarrow x_{0}$ exponentially quickly as $n \rightarrow+\infty$. The analytic function $g(\omega)$ is now given by $g(\omega)=\sum_{j=1}^{\infty} e^{-i j \omega}\left(v\left(x_{j}\right)-v\left(x_{0}\right)\right)$. Similarly for $x_{0}$ periodic. The argument is the same as before with perturbations made at $x_{1}$.

\section{Exponentially and slowly mixing nonuniformly hyperbolic systems}

In this section, we indicate how our results extend to more general classes of dynamical systems, such as those described in [1, 21, 23, 24].

For noninvertible maps, parts (i) and (ii) of Theorem 1.1 require only that the Perron-Frobenius operator $L$ is quasicompact (the spectrum consists of finitely many eigenvalues on the unit circle, and the remainder of the spectrum strictly inside the unit circle) on a suitable Banach space of observables. If this Banach space lies inside the space of continuous functions, then part (iii) of Theorem 1.1 is also valid.

For invertible maps, a preliminary argument is often required as in Section 3 to reduce to the noninvertible case. The tower construction of Young [23] satisfies all of the requirements for our results to apply. For example, planar periodic dispersing billiards and Hénon-like attractors are covered by Young's construction and hence parts (i)-(iii) of Theorems 1.1 and 1.3 hold for such systems.

Our results on nonvanishing spectra apply also in cases where the decay of correlations is subexponential. For example, consider Pomeau-Manneville intermittency maps $[18,13]$ where $f(x) \approx x+x^{1+\alpha}$. These maps have the decay of correlations rate $\rho(k) \approx k^{-\left(\frac{1}{\alpha}-1\right)}[9]$. The Wiener-Khinchin theorem applies for $0<\alpha<\frac{1}{2}$, and the power spectrum is continuous but at most finitely many times differentiable. Nevertheless, the iterates of $L^{k} \hat{v}$ remain summable $[9,24]$ and hence our criterion $(2.3)$ for $S(\omega)=0$ holds. Moreover, the function $g$ in (3.1) is analytic as before; analyt- 
icity of $g$ depends on exponential convergence along stable and unstable manifolds of the periodic point $x_{0}$ (excluding the indifferent fixed point at 0 ), and so is unaffected by the slow convergence of $L$. It follows that the spectrum is again bounded away from zero for typical Hölder observables. The same result holds for the general class of nonuniformly hyperbolic systems with polynomial, but summable, decay of correlations considered in [24].

\section{Numerical results}

In this paper we have obtained results on the analytic and nonvanishing nature of broadband power spectra characteristic of deterministic chaos. In Figs. 1-4 we provide numerical simulations for the logistic map illustrating our results. We consider the parameter value $a=3.757$ where the dynamics is chaotic but nonmixing. Indeed the attractor consists of 4 intervals permuted cyclically by $f$, and $f^{4}$ is mixing on each of these intervals. In particular, there are removable singularities at $\omega=k \pi / 2$, $k=0, \ldots, 4$ and Figure 3 shows the convergence to the removable singularity at $\omega=\pi / 2$. There is an additional approximate cycling of period 8 yielding peaks at $\omega=k \pi / 4, k=1,3,5,7$. Figure 4 confirms that these additional peaks are nonsingular. Froyland [5] has verified numerically that the Perron-Frobenius operator $L$ has precisely 4 eigenvalues on the unit circle at 4 'th roots of unity and a further 4 eigenvalues close to the unit circle near the remaining 8'th roots of unity. (Thus one may use the convergence of the spectrum at such a nonsingular peak as an indicator for almost invariant sets. Such eigenvalues may slow down the convergence of $L$ and hence are obstructions to rapid convergence of the power spectrum.)

\section{A Power spectra for nonmixing systems}

As mentioned in Remark 1.2(a), it is well-known that the Wiener-Khinchin theorem relates smoothness of the power spectrum to decay of correlations of $f^{q}$ when $q=1$. For completeness, we now state and prove the analogous result for $q \geq 2$.

Let $f: X \rightarrow X$ be a discrete dynamical system with ergodic invariant measure $\mu$. Suppose that $f$ is mixing up to a $q$ cycle, so $X=X_{1} \cup \cdots \cup X_{q}$ where $f\left(X_{i}\right)=X_{i+1}$ (indices computed $\bmod q$ ) and $f^{q}: X_{i} \rightarrow X_{i}$ is mixing for $i=1, \ldots, q$.

Let $v: X \rightarrow \mathbb{R}$ be a square-integrable observable. For $q \geq 2$, there is no decay of correlations (since $f: X \rightarrow X$ is not mixing). Nevertheless, there is a fairly natural notion of summability (and moreover exponential decay) up to a $q$ cycle. For each fixed $\omega \in[0,2 \pi]$, define $v_{q, \omega}: X \rightarrow \mathbb{C}$ by $v_{q, \omega}=\sum_{\ell=0}^{q-1} e^{i \ell \omega} v \circ f^{\ell}$. Let $\mu_{\ell}=\left.q \mu\right|_{X_{\ell}}$ be 
the normalised restriction of $\mu$ to $X_{\ell}, \ell=1, \ldots, q$. Define

$$
\rho_{q, \omega}(k)=\frac{1}{q} \sum_{\ell=1}^{q}\left\{\int_{X_{\ell}} v_{q, \omega} \circ f^{q k} \bar{v}_{q, \omega} d \mu_{\ell}-\left|\int_{X_{\ell}} v_{q, \omega} d \mu_{\ell}\right|^{2}\right\}, \quad k \in \mathbb{Z} .
$$

(If $f$ is noninvertible, then we interpret $\rho(k), k$ negative, to be

$$
\left.\rho_{q, \omega}(k)=\bar{\rho}_{q, \omega}(-k)=\frac{1}{q} \sum_{\ell=1}^{q}\left\{\int_{X_{\ell}} v_{q, \omega} \bar{v}_{q, \omega} \circ f^{-q k} d \mu_{\ell}-\left|\int_{X_{\ell}} v_{q, \omega} d \mu_{\ell}\right|^{2}\right\} .\right)
$$

Definition A.1 We say that the autocorrelations of $v$ are summable up to a $q$ cycle if the series $\sum_{k=0}^{\infty}\left|\rho_{q, \omega}(k)\right|$ is convergent for each $\omega \in[0,2 \pi]$.

The autocorrelations decay exponentially up to a $q$ cycle if there exist constants $C \geq 1, \tau \in(0,1), \epsilon>0$, independent of $\omega$ such that

$$
\left|\rho_{q, \omega+i \sigma}(k)\right| \leq C \tau^{k} \text {, for all } k \geq 0, \omega \in[0,2 \pi], \sigma \in[-\epsilon, \epsilon] .
$$

Theorem A.2 Suppose that $f: X \rightarrow X$ is mixing up to a $q$ cycle and that the square-integrable observable $v: X \rightarrow \mathbb{R}$ has summable autocorrelations up to a $q$ cycle. Then

$$
S(\omega)=\sum_{k=-\infty}^{\infty} e^{i k q \omega} \rho_{q, \omega}(k),
$$

for all $\omega \neq 2 \pi j / q, j=0,1, \ldots, q$.

In particular, if $v$ has exponentially decaying autocorrelations up to a $q$ cycle, then $S(\omega)$ is analytic except possibly for finitely many removable singularities at $\omega=2 \pi j / q$, $j=0,1, \ldots, q$.

Proof Suppose that $\omega \neq 2 \pi j / q, j=0,1, \ldots, q$. By the definition of $v_{q, \omega}$,

$$
\begin{aligned}
\int_{X}\left|\sum_{j=0}^{q n-1} e^{i j \omega} v \circ f^{j}\right|^{2} d \mu & =\int_{X}\left|\sum_{j=0}^{n-1} e^{i j q \omega} v_{q, \omega} \circ f^{j q}\right|^{2} d \mu \\
& =\sum_{j, k=0}^{n-1} e^{i(j-k) q \omega} \int_{X} v_{q, \omega} \circ f^{(j-k) q} \bar{v}_{q, \omega} d \mu \\
& =\sum_{j, k=0}^{n-1} e^{i(j-k) q \omega}\left\{\rho_{q, \omega}(j-k)+\frac{1}{q} \sum_{\ell=1}^{q}\left|\int_{X_{\ell}} v_{q, \omega} d \mu_{\ell}\right|^{2}\right\} \\
& =\sum_{j=-(n-1)}^{n-1}(n-|j|) e^{i j q \omega} \rho_{q, \omega}(j)+R_{\omega, n}
\end{aligned}
$$

where

$$
R_{\omega, n}=\frac{1-\cos n q \omega}{1-\cos q \omega} \frac{1}{q} \sum_{\ell=1}^{q}\left|\int_{X_{\ell}} v_{q, \omega} d \mu_{\ell}\right|^{2}
$$


Hence we can write

$$
\int_{X}\left|\sum_{j=0}^{q n-1} e^{i j \omega} v \circ f^{j}\right|^{2} d \mu=s_{1}+\cdots+s_{n}+R_{\omega, n}
$$

where $s_{k}=\sum_{j=-(k-1)}^{k-1} e^{i j q \omega} \rho_{q, \omega}(j)$. Since $R_{\omega, n}$ is bounded (for each fixed $\left.\omega\right), S(\omega)=$ $\lim _{n \rightarrow \infty}\left(s_{1}+\cdots+s_{n}\right) / n$ is the Cesàro limit of the sequence $\sum_{j=-(n-1)}^{n-1} e^{i j q \omega} \rho_{q, \omega}(j)$. The summability condition on $\rho_{q, \omega}$ guarantees that the series $\sum_{j=-\infty}^{\infty} e^{i j q \omega} \rho_{q, \omega}(j)$ is convergent and hence coincides with the Cesàro limit, proving the first statement of the theorem. The second statement is immediate.

Remark A.3 When $q=1$, the first statement of the theorem is the usual WienerKhinchin theorem and $\rho_{1, \omega}(k)=\rho(k)$ is independent of $\omega$. Note that to obtain continuity of the power spectrum for $q \geq 2$, it is necessary to assume that the series $\sum_{j=0}^{\infty}\left|\rho_{q, \omega}(j)\right|$ is uniformly convergent.

Acknowledgments The research of GAG was partly supported by ARC grant DP0452147. The research of IM was partly supported by EPSRC grant EP/D055520/1 and by a Leverhulme Research Fellowship. IM is very grateful to the University of Sydney for its hospitality.

\section{References}

[1] V. Baladi. Positive Transfer Operators and Decay of Correlations. Advanced Series in Nonlinear Dynamics 16, World Scientific, 2000.

[2] M. Benedicks and L. Carleson. On iterations of $1-a x^{2}$ on $(-1,1)$. Ann. of Math. $122(1985)$ 1-25.

[3] R. Bowen. Equilibrium states and the ergodic theory of Anosov diffeomorphisms. Lecture Notes in Mathematics 470, Springer, Berlin, 1975.

[4] P. Collet and J.-P. Eckmann. Positive Liapunov exponents and absolute continuity for maps of the interval. Ergodic Theory Dynam. Systems 3 (1983) 13-46.

[5] G. Froyland. Private communication.

[6] P. Gaspard. Chaos, Scattering and Statistical Mechanics. Cambridge University Press, Cambridge, 1998. 
[7] J. P. Gollub and H. L. Swinney. Onset of turbulence in a rotating fluid. Phys. Rev. Lett. 35 (1975) 927-930.

[8] M. I. Gordin. The central limit theorem for stationary processes. Soviet Math. Dokl. 10 (1969) 1174-1176.

[9] H. Hu. Decay of correlations for piecewise smooth maps with indifferent fixed points. Ergodic Theory Dynam. Systems 24 (2004) 495-524.

[10] M. Jakobson. Absolutely continuous invariant measures for one-parameter families of one-dimensional maps. Comm. Math. Phys. 81 (1981) 39-88.

[11] N. G. van Kampen. Stochastic Processes in Physics and Chemistry. NorthHolland, Amsterdam, 2003.

[12] G. Keller and T. Nowicki. Spectral theory, zeta functions and the distribution of periodic points for Collet-Eckmann maps. Comm. Math. Phys. 149 (1992) 31-69.

[13] C. Liverani, B. Saussol and S. Vaienti. A probabilistic approach to intermittency. Ergodic Theory Dynam. Systems 19 (1999) 671-685.

[14] M. Lyubich. Almost every real quadratic map is either regular or stochastic. Ann. of Math. 156 (2002) 1-78.

[15] I. Melbourne and M. Nicol. Statistical properties of endomorphisms and compact group extensions. J. London. Math. Soc. 70 (2004) 427-446.

[16] M. Nicol, I. Melbourne and P. Ashwin. Euclidean extensions of dynamical systems. Nonlinearity 14 (2001) 275-300.

[17] W. Parry and M. Pollicott. Zeta Functions and the Periodic Orbit Structure of Hyperbolic Dynamics. Astérique 187-188, Société Mathématique de France, Montrouge, 1990.

[18] Y. Pomeau and P. Manneville. Intermittent transition to turbulence in dissipative dynamical systems. Comm. Math. Phys. 74 (1980) 189-197.

[19] D. Ruelle. Resonances of chaotic dynamical systems. Phys. Rev. Lett. 56 (1986) 405-407.

[20] Y. G. Sinai. Gibbs measures in ergodic theory. Russ. Math. Surv. 27 (1972) 21-70. 
[21] M. Viana. Stochastic dynamics of deterministic systems. Col. Bras. de Matemática, 1997.

[22] L.-S. Young. Decay of correlations for certain quadratic maps. Comm. Math. Phys. 146 (1992) 123-138.

[23] L.-S. Young. Statistical properties of dynamical systems with some hyperbolicity. Ann. of Math. 147 (1998) 585-650.

[24] L.-S. Young. Recurrence times and rates of mixing. Israel J. Math. 110 (1999) $153-188$.

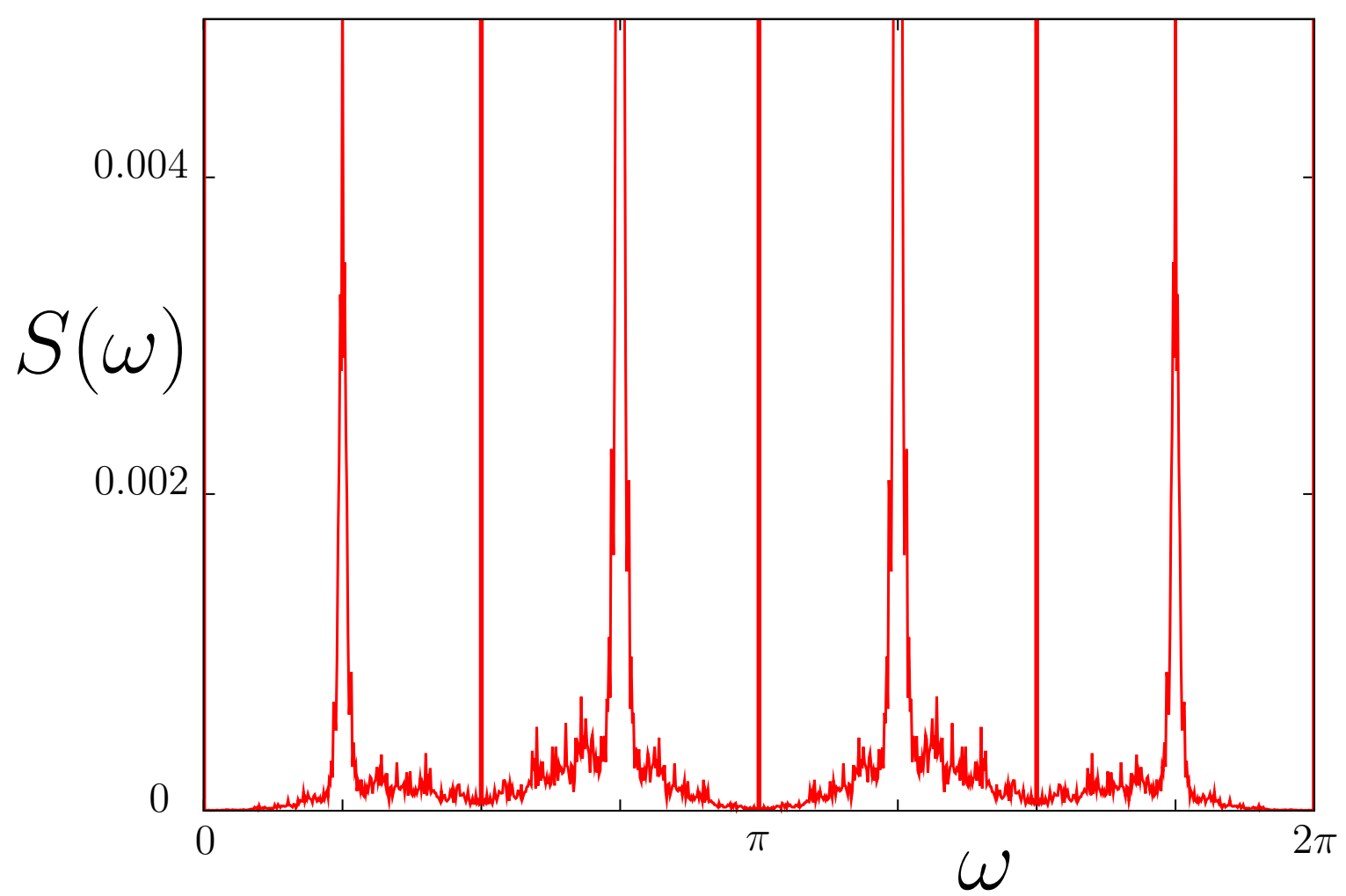

Figure 1: The power spectrum $S(\omega)$ for the logistic map with $a=3.757$ computed using the mean square displacement along an orbit of $200 K$ iterates. The dynamics is chaotic but non-mixing; the invariant measure is supported on 4 distinct intervals which the orbit visits in succession. The cycling is confirmed by peaks at $\pi / 2, \pi$ and $3 \pi / 2$ where $S(\omega)=\infty$. The numerically computed values are $S(\pi / 2) \approx 50$, $S(\pi) \approx 900$. In contrast, the spectrum is finite at the approximate but nonsingular peaks at $k \pi / 4, k=1,3,5,7$ with values $S(\pi / 4) \approx 0.006$ and $S(3 \pi / 4) \approx 0.015$. 


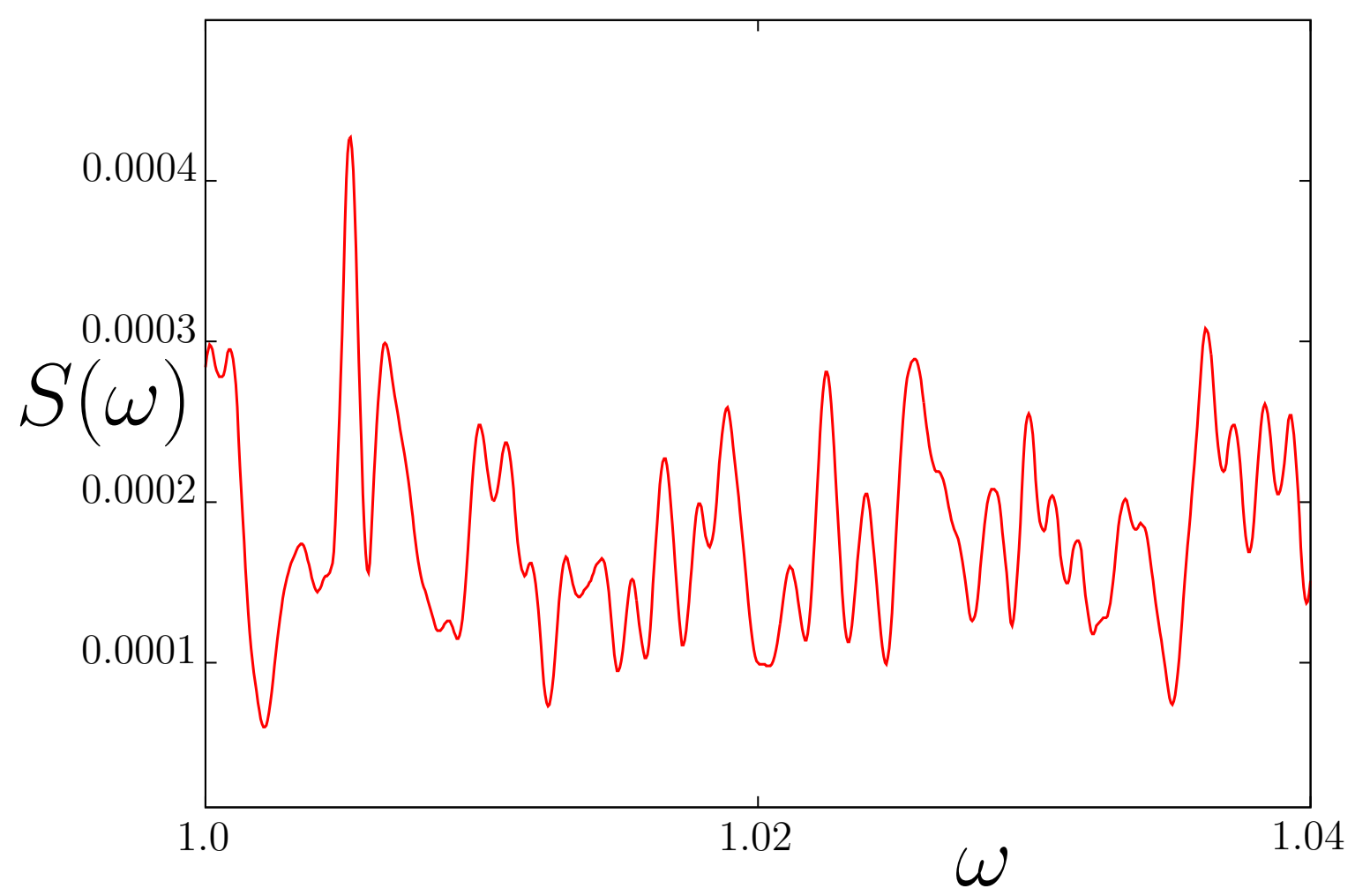

Figure 2: As in Fig. 1, but now a small interval of $\omega$ is shown to illustrate that $S(\omega)$ is indeed analytic and nonzero. 


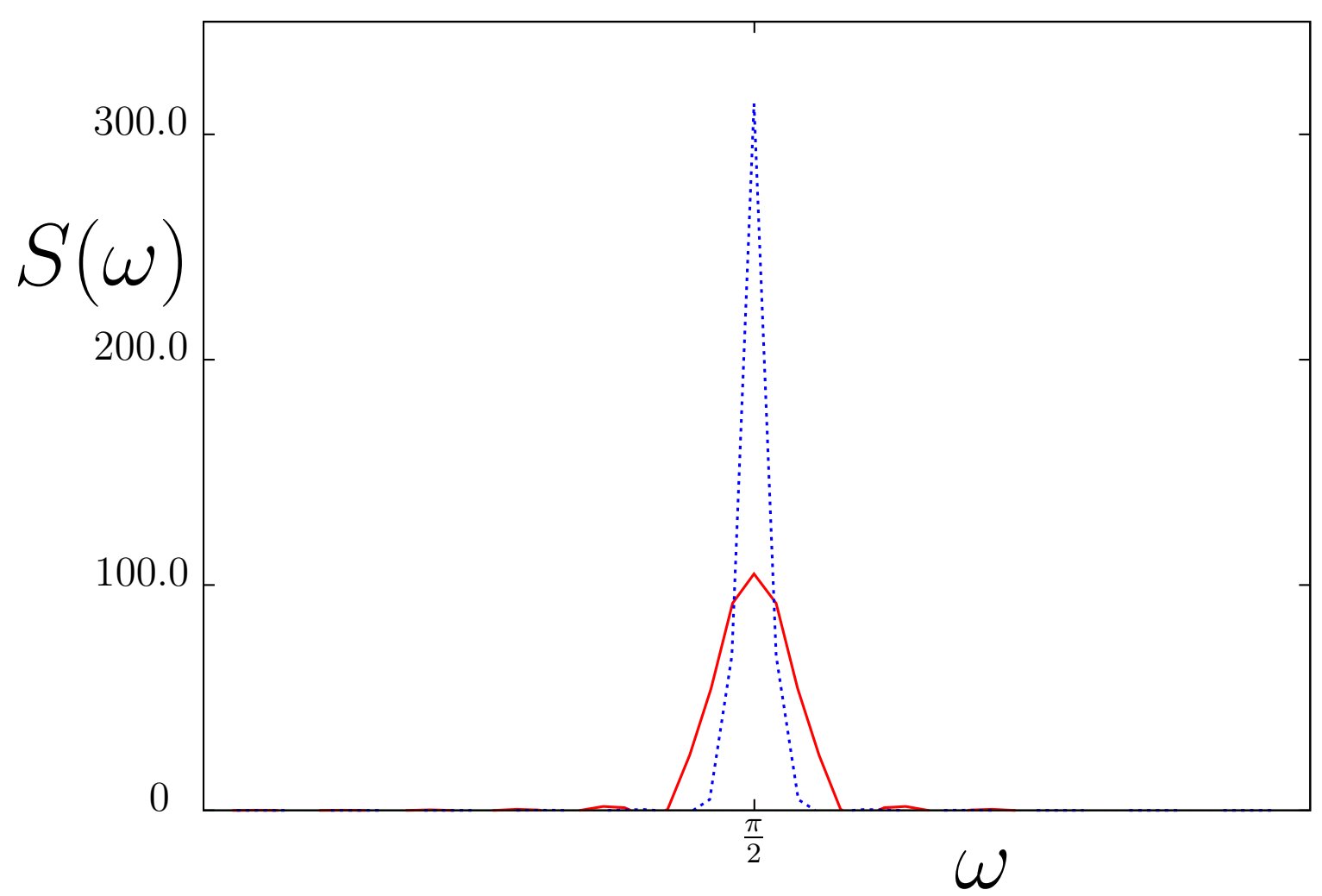

Figure 3: As in Fig. 1, but now a zoom in on the removable singularity at $\omega=\pi / 2$ (continuous lines $500 \mathrm{~K}$ iterates, dashed lines $2000 \mathrm{~K}$ iterates). 


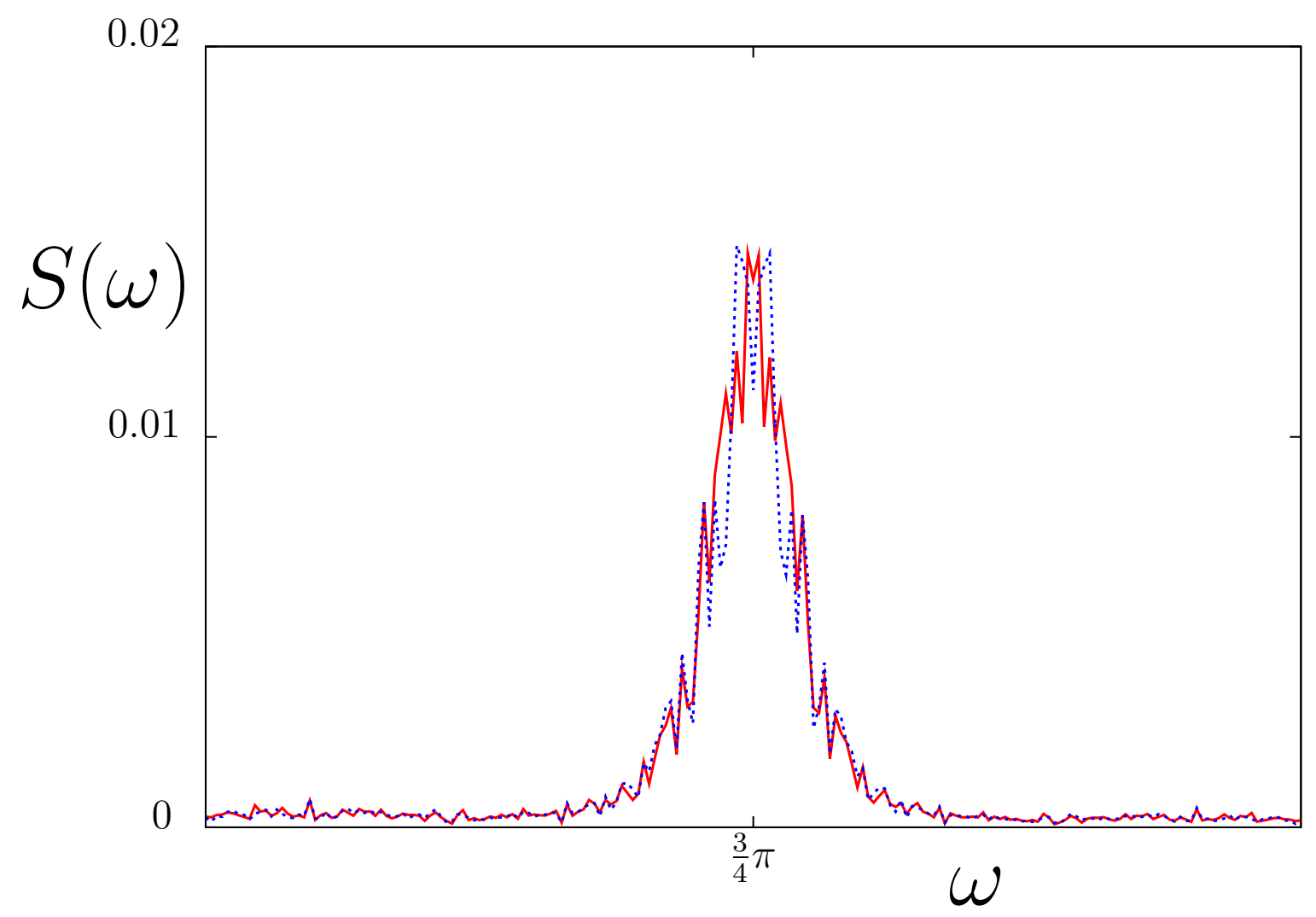

Figure 4: As in Fig. 3, but now a zoom in on the nonsingular peak at $\omega=3 \pi / 4$. 